\title{
NOUVELLE
}

\section{RelA, un nouvel acteur dans la régulation de l'activité de RelB}

Émilie Jacque, Véronique Baud

\author{
Institut Cochin, Département d'Hématologie \\ Inserm U567, CNRS UMR 8104, \\ Université Paris-Descartes, \\ Maternité Port-Royal, \\ 123, boulevard de Port-Royal, 75014, Paris, France. \\ vbaud@cochin.inserm.fr
}

> Vingt ans après leur découverte, l'intérêt croissant que suscitent les facteurs de transcription NF- $\kappa B$ émane de la multiplicité des réponses cellulaires auxquelles ces facteurs sont associés. Ils jouent en effet un rôle clé dans la réponse immune, inflammatoire, proliférative et apoptotique, mais aussi dans la tumorigenèse [1-4]. Ainsi, le dérèglement de l'activité de ces facteurs, via la production de formes anormales de $N F-\kappa B$, ou une régulation défectueuse de leurs activités transcriptionnelles, est retrouvée de manière récurrente dans de nombreuses formes de cancers telles que les leucémies chroniques lymphoïdes et lymphocytaires à cellules B et de nombreux lymphomes [4]. Plus récemment, il a été mis en évidence que les facteurs NF-KB participent également au développement des tumeurs en activant des gènes de résistance à l'apoptose, rendant ainsi certaines tumeurs résistantes aux traitements chimiothérapiques et radiothérapiques [5]. Il apparaît donc clairement que l'élucidation des mécanismes moléculaires permettant l'activation de ces facteurs est indispensable à la conception de thérapeutiques futures fondées sur le contrôle de l'activité transcriptionnelle de ces protéines.

La famille NF- $\kappa B$ compte cinq membres chez les vertébrés: NF- $\kappa B l$ ( $p 50$ et son précurseur $p 105), N F-\kappa B 2$ ( $p 52$ et son précurseur $p 100), c-\operatorname{Rel}, \operatorname{Rel} A(p 65)$ et RelB [6]. Ces protéines s'associent en une collection variée d'homo- et d'hétérodimères, dont l'activité transcriptionnelle est régulée par deux voies principales. La première, plus connue sous le terme de voie d'activation canonique de $N F-\kappa B$, s'applique principalement aux dimères RelA/p50 [7]. Sous leur forme inactive, les dimères RelA/ p50 sont retenus dans le cytoplasme par leur association avec les protéines inhibitrices $\mid \kappa B$. En réponse à une grande variété de stimuli comme les cytokines pro-inflammatoires (TNF $\alpha, \mathrm{IL}-1$ ), lipopolysaccharides bactériens (LPS), virus, protéines virales, ARN double brin, esters de phorbol, agents génotoxiques et radiations, les $\mid \kappa B$ sont rapidement phosphorylées, ubiquitinylées et, finalement, dégradées. Les dimères RelA/p50 ainsi libérés passent alors dans le noyau et activent la transcription de gènes cibles spécifiques [7]. Plus récemment, une seconde voie d'activation de $N F-\kappa B$, qui s'applique principalement à RelB, a été caractérisée. Cette voie, maintenant appelée voie alternative d'activation de $N F-\kappa B$, repose sur la protéolyse induite par différents membres de la superfamille du TNF (tumor necrosis factor) (lymphotoxine $\beta$, BAFF) de la protéine p100, l'inhibiteur principal de RelB, et aboutit à la libération, la translocation nucléaire et la liaison à l'ADN des dimères $\operatorname{RelB/p50~et~RelB/p52~[8,9].~}$

Il est important de noter que le TNF $\alpha$ et la lymphotoxine $\beta$, bien que faisant toutes deux partie de la superfamille du TNF, n'induisent pourtant pas les mêmes cascades de signalisation de NF$\kappa \mathrm{B}$ [9]. Ainsi, le TNF $\alpha$ est un inducteur de la voie canonique (RelA) et non de la voie alternative de $N F-\kappa B$ (RelB). Quelle n'a donc pas été notre surprise quand nous avons observé que la stimulation par le TNF $\alpha$ induit pourtant l'accumulation nucléaire de RelB dans les fibroblastes embryonnaires murins (MEF) [10]! Néanmoins, contrairement à RelA, l'accumulation nucléaire de RelB induite par le TNF $\alpha$ n'aboutit pas à une augmentation de sa liaison à l'ADN, suggérant l'existence de mécanismes de régulation de la liaison à l'ADN de RelB dans le noyau de ces cellules. Le TNF $\alpha$ étant l'inducteur par excellence de la voie canonique et donc de l'activité de RelA, nous avons entrepris de décrypter le rôle joué par RelA dans la régulation de RelB en réponse au TNF $\alpha$.

Notre étude a ainsi mis en évidence que la stimulation par le TNF $\alpha$ induit l'association de RelA avec RelB dans le noyau des MEF et que la séquestration de RelB dans les hétérodimères RelA/RelB bloque sa liaison à I'ADN [10]. Nous avons également démontré que la sérine-276 de RelA, dont la phosphorylation est induite par le TNF $\alpha$ in vivo, est cruciale pour la formation du complexe RelA/ RelB ainsi que pour l'inhibition subséquente de la liaison à I'ADN de RelB (Figure 1).

Il était important, dans une seconde étape, de déterminer si l'effet inhibiteur de RelA sur la liaison à I'ADN de RelB pouvait affecter la transcription de gènes cibles de la famille NF-KB. Nous avons ainsi identifié plusieurs gènes cibles, parmi lesquels le gène anti-apoptotique $B C l-x L$, dont l'expression est fortement induite par le TNF $\alpha$ dans les fibroblastes déficients en RelA alors qu'une telle induction n'est pas observée dans les 


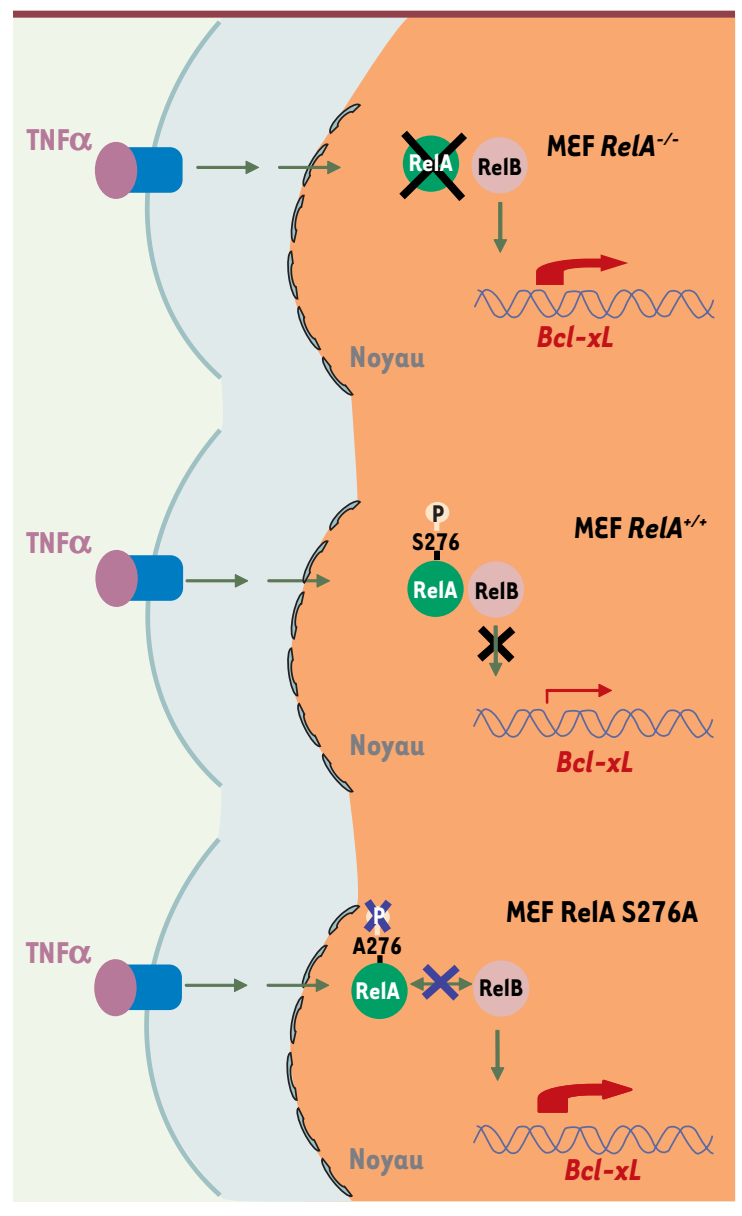

Figure 1. Modèle du mécanisme de régulation de RelB par RelA en réponse au TNF $\alpha$. Dans les fibroblastes embryonnaires murins sauvages (MEF Rel $A^{+/+}$) (milieu), la stimulation par le TNF $\alpha$ induit l'association de RelA avec RelB dans le noyau, ce qui conduit à l'inhibition de la liaison à l'ADN de RelB ainsi qu'à une faible induction de l'expression de certains gènes cibles de NF- $K B$ dont le gène antiapoptotique $B c l-x L$. En revanche, dans les fibroblastes déficients en RelA (MEF RelA ${ }^{-/}$) (haut), RelB est libre de se lier au promoteur de $B c l-x L$ et d'induire fortement son expression en réponse au TNF $\alpha$. Le rôle crucial de la phosphorylation de la sérine-276 de RelA dans ce mécanisme est également illustré (bas). La mutation de la sérine-276 de RelA en alanine (MEF RelA S276A) réduit très fortement l'interaction de RelA avec RelB, permettant ainsi à RelB de se lier à l'ADN et d'activer fortement la transcription de $B C l-x L$. cellules sauvages [10]. Cette observation montre bien que RelA, jusqu'alors décrit uniquement comme un activateur transcriptionnel, pouvait également exercer des fonctions répressives servant à limiter la transcription de certains gènes cibles de NF-KB. Nous avons ensuite démontré par interférence ARN que RelB contrôle l'augmentation de l'expression de $\mathrm{Bcl}$ $x L$ induite par le TNF $\alpha$ en l'absence de RelA, et nous avons mis en évidence par immunoprécipitation de chromatine que RelB se lie au promoteur de $B c l-x L$ [10]. Ces données révèlent donc que l'activité répressive de RelA sur l'expression de $\mathrm{Bcl}-\mathrm{xL}$ s'exerce bien via la régulation de l'activité de RelB. La sérine276 de RelA étant cruciale pour la formation du complexe RelA/RelB et le blocage de la liaison à I'ADN de RelB, l'ensemble de nos résultats suggère que l'hétérodimérisation de RelA avec RelB permet de limiter l'expression de certaines cibles transcriptionnelles de Rel $B$, telles que le gène anti-apoptotique $B c l-x L$ (Figure 1). $\diamond$

\section{En conclusion}

Nos travaux ont permis de mettre à jour un nouveau mécanisme nucléaire de régulation de l'activité de RelB, qui repose sur son association avec RelA, mais également de démontrer que cette régulation croisée entre RelA et RelB est de toute première importance dans le contrôle de l'expression de gènes cibles de NF- $\kappa B$. La découverte de ce mécanisme ouvre maintenant de nouveaux champs d'investigation du rôle de RelB dans le contrôle de la prolifération et de l'apoptose cellulaires, mais aussi dans la progression tumorale. $\diamond$

RelA, a new player"

in the regulation of RelB activity

\section{RÉFÉRENCES}

1. Baeuerle PA, Henkel T. Function and activation of NF$\kappa B$ in the immune system. Annu Rev Immunol 1994; $12: 141-79$.

2. Barnes PJ, Karin M. Nuclear factor- $\kappa B$ : a pivotal transcription factor in chronic inflammatory diseases. N EnglJ Med 1997 ; 336 : 1066-71.

3. Karin $M$, Lin A. NF- $K B$ at the crossroads of life and death. Nat Immunol $2002 ; 3: 221-7$.

4. Rayet $B$, Gélinas $C$. Aberrant rel/NF- $\kappa B$ genes and activity in human cancer. Oncogene 1999 ; $18: 6938-47$

5. Wang Cy, Cusack JC, Liu R, Baldwin AS. Control of inducible chemoresistance : enhanced anti-tumor therapy through increased apoptosis by inhibition of NF-KB. Nat Med 1999; 5 : 412-7.

6. Ghosh S, May MJ, Kopp EB. NF-KB and Rel proteins : evolutionarily conserved mediators of immune responses. Annu Rev Immunol 1998; 16 : 225-60.

7. Karin M, Ben-Neriah Y. Phosphorylation meets ubiquitination : the control of NF- $\kappa B$ activity. Annu Rev Immunol $2000 ; 18$ : 621-63.

8. Pomerantz JL, Baltimore D. Two pathways to NF- $\kappa B$. Mol Cell $2003 ; 10: 693-5$.

9. Derudder $\varepsilon$, Dejardin $\varepsilon$, Pritchard LL, et al. RelB/p50 dimers are differentially regulated by TNF- $\alpha$ and lymphotoxin- $ß$ receptor activation : critical roles for p100. J Biol Chem 2003 ; 278 : 23278-84.

10. Jacque $\varepsilon$, Tchenio T, Piton $G$, et al. RelA repression of RelB activity induces selective gene activation downstream of TNFR. Proc Natl Acad Sci USA 2005; 102 : $14635-40$.

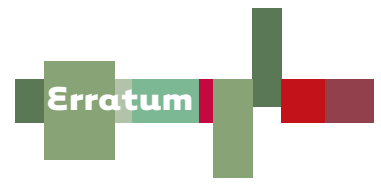

Dans $\mathrm{m} / \mathrm{s} \mathrm{n}^{\circ} 1$, vol. 22, janvier 2006, le titre anglais de la Nouvelle de Lluis Quintana-Murci (p. 29-31) «Immunité innée et sélection naturelle : le paradigme de la région génomique DC-SIGN/L-SIGN » est erroné. II faut lire : Innate immunity and natural selection : the paradigm of the DC-SIGN/L-SIGN genomic region. 